\title{
Spontaneous and evoked intracellular calcium transients in donor-derived myocytes following intracardiac myoblast transplantation
}

\author{
Michael Rubart, Mark H. Soonpaa, Hidehiro Nakajima, and Loren J. Field \\ Wells Center for Pediatric Research and Krannert Institute of Cardiology, Indiana University School of Medicine, Indianapolis, Indiana, USA
}

\begin{abstract}
Skeletal myoblast transplantation is a potential treatment for congestive heart failure. To study the functional activity of both donor and host myocytes following transplantation, skeletal myoblasts expressing an enhanced green fluorescent protein (EGFP) transgene were transplanted into hearts of nontransgenic recipients, and changes in intracellular calcium concentration $\left(\left[\mathrm{Ca}^{2+}\right]_{i}\right)$ were monitored in donor and host cells. While the vast majority of donor-derived myocytes were observed to be functionally isolated from the host myocardium, a small population of donor myocytes exhibited action potential-induced calcium transients in synchrony with adjacent host cardiomyocytes. In many cases, the durations of these $\left[\mathrm{Ca}^{2+}\right]_{\mathrm{i}}$ transients were heterogeneous compared with those in neighboring host cardiomyocytes. In other studies, EGFP-expressing donor myoblasts were transplanted into the hearts of adult transgenic recipient mice expressing a cardiomyocyte-restricted $\beta$-gal reporter gene. A small population of myocytes was observed to express both reporter transgenes, indicating that the transplanted myoblasts fused with host cardiomyocytes at a very low frequency. These cells also expressed connexin 43, a component of gap junctions. Thus engraftment of skeletal myoblasts generated spatial heterogeneity of $\left[\mathrm{Ca}^{2+}\right]_{i}$ signaling at the myocardial/skeletal muscle interface, most likely as a consequence of fusion events between donor myoblasts and host cardiomyocytes.
\end{abstract}

\section{Introduction}

Numerous animal studies have demonstrated that skeletal myoblasts differentiate into myocytes and form stable grafts following cellular transplantation into normal or injured myocardium (1). Transplantation into injured hearts appeared to limit adverse remodeling, and in some cases resulted in either regional or global improvement in left ventricular function. The absence of skeletal myocyte-cardiomyocyte gap junctional complexes (2), the presence of voltage-dependent recruitment of contractile activity (3), and the absence of dye transfer and gross electromechanical coupling in explanted tissue (4) all suggested that the donor-derived myocytes were not functionally coupled to the host myocardium. The improved cardiac function observed following transplantation into injured hearts thus was likely due to secondary events, for example, enhanced angiogenesis and/ or altered scar elastic properties due to the presence of donor cells. Indeed, several recent clinical studies showed that transplanted autologous myoblasts formed stable myocyte grafts and improved global cardiac function in humans (5-7).

The ability to examine the functional consequences of donorhost interactions at the cellular level in situ would likely contribute to a more basic understanding of how myoblast cell transplantation might impact host heart function. Recently, two-photon molecular excitation (TPME) laser scanning microscopy was used to image calcium-dependent changes in rhod-2 indicator dye fluorescence within individual cardiomyocytes in intact hearts (8). The

Nonstandard abbreviations used: ACT, chicken $\beta$-actin; $\left[\mathrm{Ca}^{2+}\right]_{i}$, intracellular calcium concentration; EGFP, enhanced green fluorescent protein; MHC, myosin heavy chain; nLAC, nuclear-localized $\beta$-gal; TPME, two-photon molecular excitation; $\mathrm{X}$-GAL, 5-bromo-4-chloro-3-indolyl- $\beta$-D-galactoside.

Conflict of interest: The authors have declared that no conflict of interest exists.

Citation for this article: J. Clin. Invest. 114:775-783 (2004)

doi:10.1172/JCI200421589. approach was subsequently used to image intracellular calcium concentration $\left(\left[\mathrm{Ca}^{2+}\right]_{\mathrm{i}}\right)$ transients in transplanted fetal cardiomyocytes expressing an enhanced green fluorescent protein (EGFP) reporter transgene (9). The use of TPME with EGFP-expressing skeletal myoblasts might constitute a useful approach to monitor donor-derived myocyte and host cardiomyocyte function following cellular transplantation. Accordingly, in this study skeletal myoblasts were isolated from transgenic mice carrying a ubiquitously expressed EGFP reporter gene (10). The EGFP-expressing myoblasts were transplanted into hearts of adult nontransgenic recipients, and TPME laser scanning microscopy was used to monitor spontaneous or stimulation-induced $\left[\mathrm{Ca}^{2+}\right]_{\mathrm{i}}$ transients in both host and donor myocytes. $\left[\mathrm{Ca}^{2+}\right]_{\mathrm{i}}$ transients were observed to occur synchronously in host cardiomyocytes, but were typically absent in the donor-derived myocytes. Quite unexpectedly, however, a small fraction of the donor-derived myocytes along the graft/host border exhibited $\left[\mathrm{Ca}^{2+}\right]_{i}$ transients in synchrony with neighboring host cardiomyocytes. In many cases the durations of the $\left[\mathrm{Ca}^{2+}\right]_{i}$ transients were heterogeneous in these donor-derived myocytes compared with host cardiomyocytes.

Additional experiments were performed wherein EGFP-expressing skeletal myoblasts were transplanted into the hearts of transgenic mice expressing a cardiomyocyte-restricted, nuclear-localized $\beta$-gal (nLAC) reporter gene (11). Histological analysis of the transplanted hearts revealed the presence of a small population of myocytes that expressed both the donor- and host-derived reporter transgene (i.e., myocytes with green fluorescent cytoplasm and nuclear $\beta$-gal activity), indicating that the transplanted skeletal myoblasts had fused with host cardiomyocytes. The prevalence and anatomical locations of these cells were very similar to those of functionally coupled, EGFP-positive myocytes identified by TPME imaging. Moreover, the presence of connexin43 immune reactivity was readily detected at junctional complexes between 
A

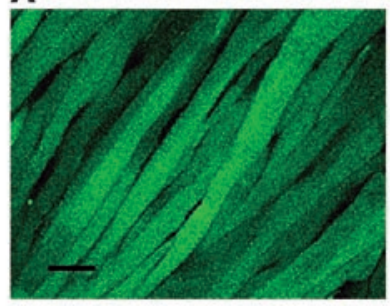

C

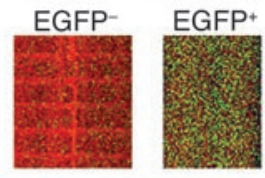

Host
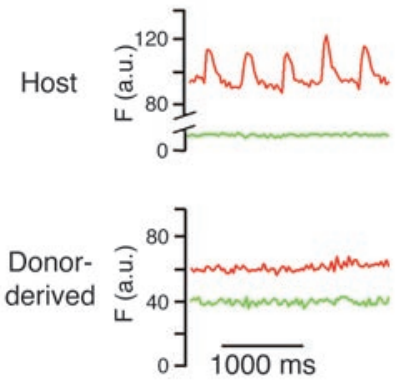

B

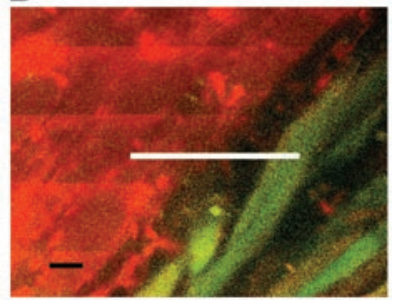

D
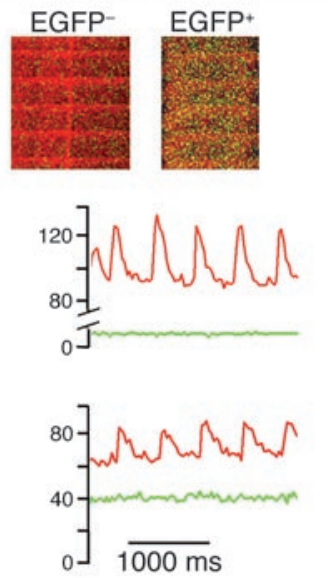

cells arising from skeletal myocyte-cardiomyocyte fusion events and the juxtaposed host cardiomyocytes. These observations are consistent with the notion that engraftment of skeletal myoblasts generates spatial heterogeneity of $\left[\mathrm{Ca}^{2+}\right]_{i}$ signaling at the myocar$\mathrm{dial} / \mathrm{skeletal}$ muscle interface, probably as a consequence of fusion events between donor myoblasts and host cardiomyocytes.

\section{Results}

Most donor-derived myocytes are functionally isolated from the host myocardium following myoblast transplantation into normal hearts. In order to monitor $\left[\mathrm{Ca}^{2+}\right]_{\mathrm{i}}$ transients in the donor-derived myocytes, it was necessary to generate skeletal myoblasts carrying a fluorescent reporter gene compatible with the TPME imaging system. Accordingly, skeletal myoblasts were isolated from mice that express an EGFP reporter gene under the regulation of the chicken $\beta$-actin (ACT) promoter, ACT-EGFP mice (10). The transgene is expressed ubiquitously in these animals, and robust fluorescence is seen in both skeletal myoblasts and skeletal myocytes. Myoblasts prepared from ACT-EGFP mice were injected directly into the left ventricle of nontransgenic adult recipients. Myoblast transplantation into uninjured hearts typically resulted in the formation of relatively large grafts comprised of well-differentiated, aligned skeletal myocytes (Figure 1A). Cell transplantation was specifically performed in uninjured hearts so as to maximize our ability to image functional interactions between donor-derived myocytes and host cardiomyocytes.

To monitor donor cell function, hearts transplanted with skeletal myoblasts were harvested, loaded with rhod-2, and imaged using TPME laser scanning microscopy. To have action potential-evoked $\left[\mathrm{Ca}^{2+}\right]_{\mathrm{i}}$ transients in the same microscopic field, initial imaging was performed at the graft/myocardium border. A representative image obtained in full-frame mode during spontaneous sinus rhythm is shown in Figure 1B. The donor-derived myocytes (which appeared yellow due to the overlay of green EGFP and red rhod-2 fluorescence) were observed to be interdigitated with the

\section{Figure 1}

Most engrafted donor-derived myocytes are functionally isolated from the host myocardium. (A) Fluorescence image of a typical intracardiac graft resulting from transplantation of ACT-EGFP myoblasts. Scale bar: $20 \mu \mathrm{m}$. (B) Full-frame TPME image of the graft/myocardium border zone of a heart following transplantation of ACT-EGFP myoblasts. Hearts were loaded with rhod-2. Host cardiomyocytes (red) and donorderived myocytes (green/yellow) are apparent. The white bar marks the position of line-scan mode data acquisition. Scale bar: $20 \mu \mathrm{m}$. (C and D) Stacked line-scan images and spatially integrated traces of the changes in rhod-2 (red) and EGFP (green) fluorescence (F) during spontaneous (C) and field stimulation-evoked (D) depolarizations from the heart depicted in $\mathbf{B}$ at 14 days following ACT-EGFP skeletal myoblast transplantation. Field stimulation was performed at $2 \mathrm{~Hz}$. Note the presence of calcium transients in the donor-derived EGFPexpressing myocyte during field stimulation of the heart, but not during sinus rhythm. a.u., arbitrary units.

host cardiomyocytes (which appeared red due to rhod-2 fluorescence). Periodic increases in rhod-2 fluorescence resulting from action potential-evoked increases in cytosolic calcium concentration are visible as ripple-like wave fronts in host cardiomyocytes, but not in donor-derived myocytes. In order to quantitate temporal changes in $\left[\mathrm{Ca}^{2+}\right]_{\text {, fluorescence signals were also recorded in }}$ line-scan mode during normal sinus rhythm. The scan line (Figure 1B, white bar) traversed a host cardiomyocyte and a juxtaposed donor-derived myocyte at a speed of $110 \mu \mathrm{m} / \mathrm{ms}$. This line was repeatedly scanned at a rate of $32 \mathrm{~Hz}$, and the resulting line scans were stacked vertically (Figure 1C, upper panel). Averaged traces of the red and green fluorescence from the host cardiomyocyte and the donor-derived myocyte were then generated from the linescan data (Figure 1C, middle and lower panels, respectively). These traces confirmed that the host cardiomyocytes exhibited transient increases in rhod-2 fluorescence, corresponding to action potential-evoked increases in $\left[\mathrm{Ca}^{2+}\right]_{i}$. In contrast, no $\left[\mathrm{Ca}^{2+}\right]_{\mathrm{i}}$ transients were detected in the donor-derived skeletal myocytes.

To exclude the possibility that this observation resulted from loss of membrane excitability or malfunction of the depolarizationinduced calcium release process in the donor-derived myocyte, calcium responses were also monitored during electrical field stimulation $(80 \mathrm{~V}, 1 \mathrm{~ms}$ duration, $2 \mathrm{~Hz})$. Under these conditions, $\left[\mathrm{Ca}^{2+}\right]_{\mathrm{i}}$ transients were no longer dependent on cell-to-cell action potential propagation. Electrical field stimulation readily evoked rhod-2 transients in both the host cardiomyocyte and the donor-derived myocyte (Figure 1D, middle and lower panels, respectively). Collectively, these data indicate that the vast majority of donor-derived myocytes present at the graft/myocardium border are not functionally coupled with the host myocardium during normal cell-to-cell electrical propagation under the imaging conditions used. However, these cells are excitable and retain the ability to raise $\left[\mathrm{Ca}^{2+}\right]_{\mathrm{i}}$ in response to membrane depolarization. Similarly, no $\left[\mathrm{Ca}^{2+}\right]_{\mathrm{i}}$ transients were detected in the central region of the skeletal myocyte grafts during spontaneous or remote point stimulation-evoked depolarizations. A total of 585 donor-derived myocytes imaged during spontaneous sinus rhythm were observed to be electrically isolated from the host myocardium. These cells were distributed among 27 independent animals and in grafts ranging from 11 to 106 days old.

Stimulation-evoked $\left[\mathrm{Ca}^{2+}\right]_{i}$ transients are similar in donor-derived myocytes in situ and in vitro. The relatively long duration of $\left[\mathrm{Ca}^{2+}\right]_{i}$ transients in field-stimulated donor-derived skeletal myocytes (see Figure 1D) was surprising, given that the duration of field stimu- 

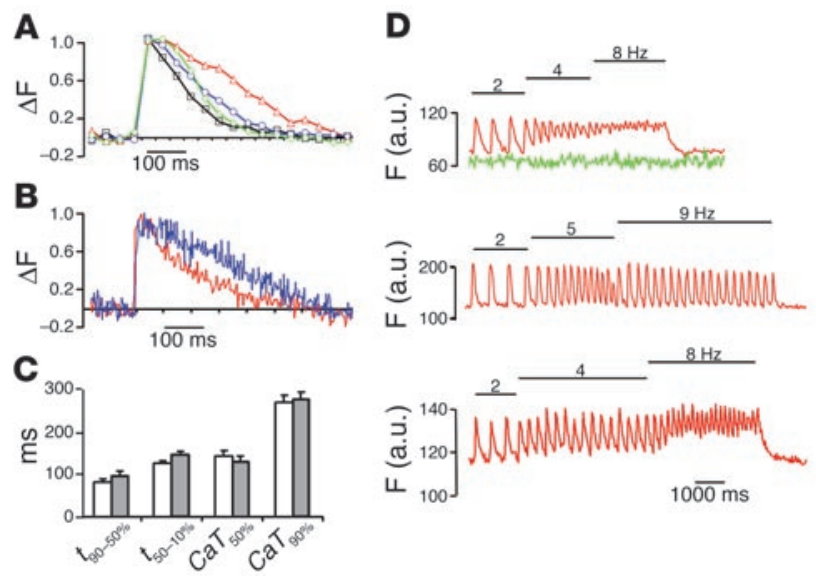

\section{Figure 2}

Donor-derived myocytes retain skeletal muscle-like functional characteristics following transplantation into the ventricular myocardium. (A) Relative changes in rhod-2 fluorescence $(\Delta F)$ as a function of time in donor-derived, EGFP-expressing myocytes (red, blue, and black traces) and a neighboring host ventricular cardiomyocyte (green trace) during electrical field stimulation $(2 \mathrm{~Hz})$. The relative changes in rhod-2 fluorescence were normalized such that 0 represents the pre-stimulus fluorescence intensity and 1 represents the peak fluorescence intensity. (B) Relative changes in rhod-2 fluorescence in in vitro-differentiated skeletal myocytes (red and blue traces) following field stimulation ( $2 \mathrm{~Hz}$; data normalized as in A). (C) Quantitative comparisons of $\left[\mathrm{Ca}^{2+}\right]_{i}$ transient parameters from donor-derived, EGFPexpressing myocytes (white bars) vs. in vitro-differentiated skeletal myocytes (gray bars) following field stimulation $(2 \mathrm{~Hz})$. Values are mean \pm SEM of 12 donor-derived, EGFP-expressing myocytes distributed among 4 hearts and from 7 in vitro-differentiated, WT skeletal myocytes after 7-10 days of culture. There were no significant differences between the groups $(P>0.05)$. (D) Spatially integrated traces of the changes in rhod-2 (red) and EGFP (green) fluorescence during field stimulation at incrementally increasing rates in a donor-derived, EGFP-expressing myocyte in situ (upper panel), in a neighboring host cardiomyocyte (middle panel), and in an in vitro-differentiated skeletal myocyte (lower panel). Note the development of tetanus in the donor-derived skeletal myocyte in situ and in the in vitro-differentiated skeletal myocyte, but not in the cardiomyocyte.

lation-induced $\left[\mathrm{Ca}^{2+}\right]_{\mathrm{i}}$ transients in adult murine skeletal muscle fibers is typically less than $100 \mathrm{~ms}$ under comparable experimental conditions (12). To further address this point, the temporal profiles of field stimulation-evoked $\left[\mathrm{Ca}^{2+}\right]_{\mathrm{i}}$ transients were quantitatively compared between donor-derived myocytes and adjacent host cardiomyocytes. Although no variability existed in the rising phase, the duration of the recovery phase differed significantly between individual donor-derived myocytes as well as between the donor-derived myocytes and their neighboring host cardiomyocytes (Figure 2A). Similar variability in recovery phase duration was seen following field stimulation of in vitro-differentiated skeletal myocytes prepared from nontransgenic mice and imaged in the absence of cytochalasin D (Figure 2B). Quantitative comparisons revealed no significant differences in $\left[\mathrm{Ca}^{2+}\right]_{i}$ transient profiles between the myoblast-derived skeletal myocytes in situ and in vitro (Figure 2C). These data also indicated that neither expression of EGFP nor the presence of cytochalasin D contributed to the heterogeneity in $\left[\mathrm{Ca}^{2+}\right]_{i}$ transient duration. Prolongation and heterogeneity in the $\left[\mathrm{Ca}^{2+}\right]_{i}$ transient recovery phase has been reported previously for skeletal myocytes following in vitro differentiation $(13,14)$. Importantly, the imaging conditions and the effects on recovery-phase kinetics were very similar to the in situ and in vitro results obtained here.

It is well known that rapid pacing in skeletal muscle results in the fusion of successive $\left[\mathrm{Ca}^{2+}\right]_{i}$ transients such that they cannot be distinguished from one another (i.e., tetanus), a property that sets it apart from cardiac muscle. To further characterize the $\left[\mathrm{Ca}^{2+}\right]_{\mathrm{i}}$ signaling phenotype in the donor-derived myocytes, hearts with cellular transplants were harvested and loaded with rhod-2, and line-scan imaging was performed during field stimulation at incrementally higher frequencies. Integrated traces of the line-scan data from a rapidly paced, engrafted donor-derived myocyte revealed that the cell developed tetanus in response to rapid stimulation (Figure 2D, upper panel). The donor-derived myocyte $\left[\mathrm{Ca}^{2+}\right]_{\mathrm{i}}$ returned to baseline levels once pacing was terminated. In contrast, $\left[\mathrm{Ca}^{2+}\right]_{\mathrm{i}}$ transient amplitudes in the host (EGFP-negative) cardiomyocyte did not develop tetanus with increased pacing rates (Figure $2 \mathrm{D}$, middle panel). As a control, in vitro-differentiated skeletal myocytes were also subjected to field stimulation at incrementally higher frequencies. As expected, the cultured skeletal myocytes developed tetanus with high-frequency stimulation (Figure 2D, lower panel). Thus, the donor-derived myocytes retained many functional properties characteristic of in vitro-differentiated cells.

Apparent functional coupling of a limited number of donor-derived myocytes at the graft/myocardium border. Quite unexpectedly, a small fraction of the donor-derived myocytes exhibited $\left[\mathrm{Ca}^{2+}\right]_{i}$ transients in synchrony with host cardiomyocytes during spontaneous sinus rhythm. These cells were localized exclusively along the graft periphery (Figure 3, A-C). Fluorescence signals were recorded in line-scan mode to quantitate temporal changes in $\left[\mathrm{Ca}^{2+}\right]_{i}$; the scan line (Figure $3, \mathrm{~A}-\mathrm{C}$; white bars) traversed at least one host cardiomyocyte (red cell) and the juxtaposed EGFPexpressing donor-derived myocyte (which appeared yellow due to the overlay of EGFP and rhod-2 fluorescence). The resulting stacked line-scan images (Figure 3, D-F) showed that rhod-2 fluorescence increased simultaneously in the EGFP-expressing donor-derived myocytes and their neighboring host cardiomyocytes (within the temporal resolution of the imaging system). The presence of synchronous $\left[\mathrm{Ca}^{2+}\right]_{i}$ transients in the donorderived myocytes and juxtaposed host cardiomyocytes strongly suggested that these cells were functionally coupled.

Spatially averaged traces from coupled donor-derived myocytes and the host cardiomyocytes along the scan lines were generated, and changes in fluorescence were normalized such that 0 represented the fluorescence value prior to $\left[\mathrm{Ca}^{2+}\right]_{\mathrm{i}}$ transient onset and 1 represented the peak fluorescence value. Superimposition of normalized $\left[\mathrm{Ca}^{2+}\right]_{i}$ transients (Figures 3, G-I) demonstrated that both the coupled donor-derived myocytes and the host cardiomyocytes exhibited rapidly rising $\left[\mathrm{Ca}^{2+}\right]_{\mathrm{i}}$ transients. In contrast, the time course of the recovery phase of the $\left[\mathrm{Ca}^{2+}\right]_{i}$ transients was heterogeneous in the coupled donor-derived myocytes. While some cells exhibited recovery phases of similar duration to those in the neighboring host cardiomyocytes (Figure 3G), others were markedly shorter (Figure 3H) or longer (Figure 3I) in duration.

On average, the values for $\left[\mathrm{Ca}^{2+}\right]_{i}$ transient duration $\left(\mathrm{CaT}_{50 \%}\right.$, $\left.\mathrm{Ca}_{90 \%}\right)$ and decay times $\left(t_{90-50 \%}, t_{50-10 \%}\right)$ were similar in the coupled donor-derived myocytes and the host cardiomyocytes during spontaneous sinus rhythm. However, given the marked heterogeneity in 

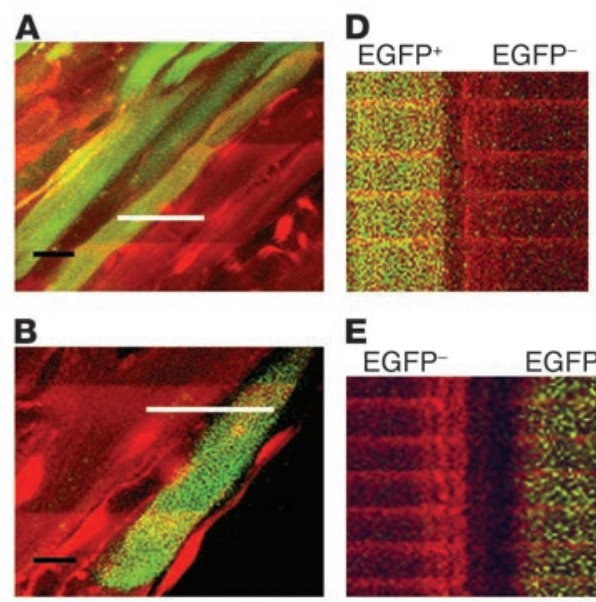

E
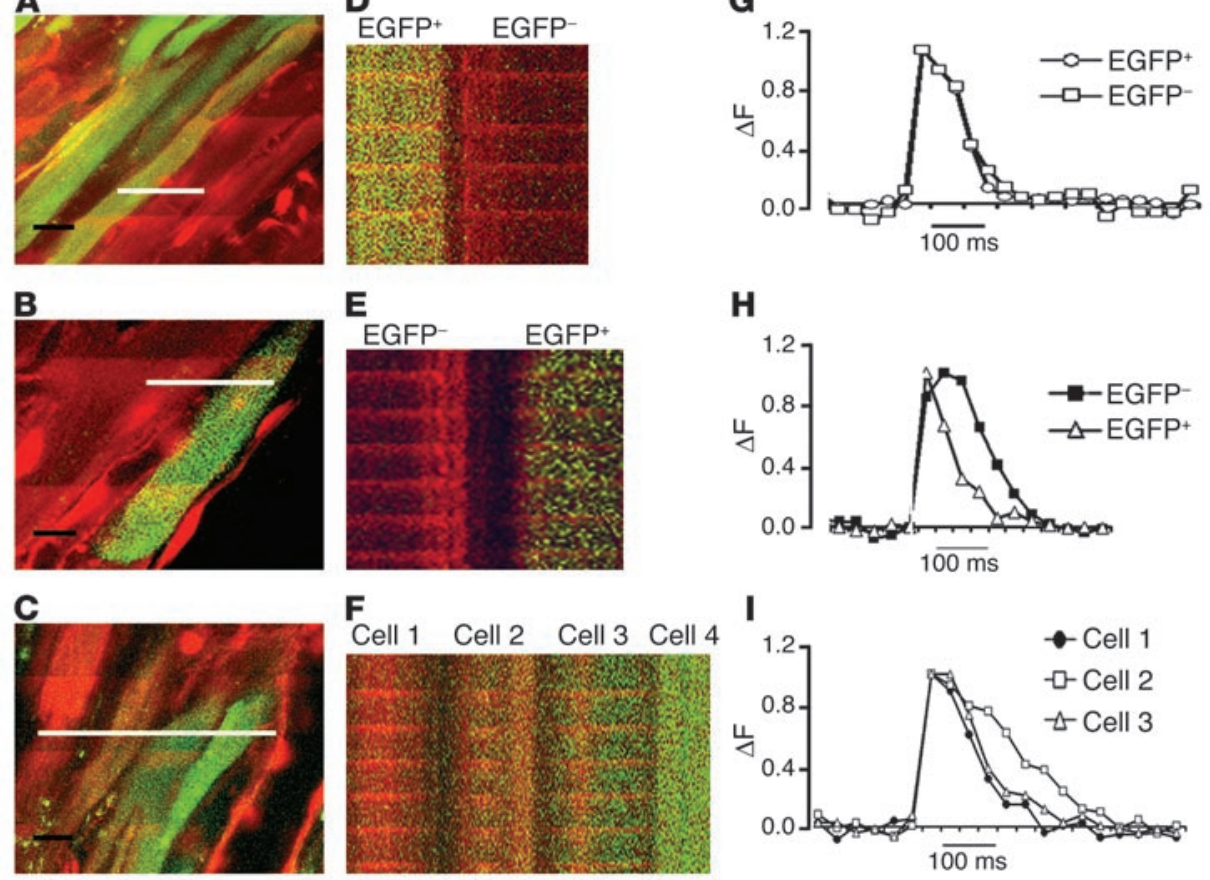

I

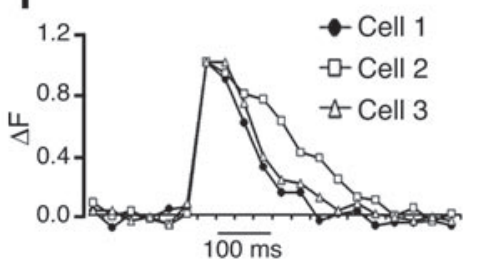

Figure 3

A small fraction of donor-derived myocytes are functionally coupled to the host myocardium. (A-C) Full frame-mode images of rhod-2-loaded hearts after transplantation of ACT-EGFP myoblasts; rhod-2 (red) and EGFP (green) signals were superimposed. White bars mark the position of the line-scan mode data acquisitions. Scale bars: $10 \mu \mathrm{m}$. (D-F) Stacked line-scan images depicting spontaneous $\left[\mathrm{Ca}^{2+}\right]_{i}$ transients in the indicated regions in panels $\mathbf{A}, \mathbf{B}$, and $\mathbf{C}$, respectively. (G-I) Superimposed tracings of spontaneous changes in rhod-2 fluorescence as a function of time from the donor-derived EGFP-positive myocyte and host EGFP-negative cardiomyocytes in $\mathbf{A}$, $\mathbf{B}$, and C, respectively. For each cell, spatially averaged changes in rhod-2 fluorescence were obtained and subsequently normalized such that 0 represents the fluorescence intensity before the $\left[\mathrm{Ca}^{2+}\right]_{i}$ transient and 1 represents the peak fluorescence intensity.

transient duration, the variances for many of the parameters measured were significantly greater in the skeletal myocytes (Table 1). Interestingly, the variability of the $\left[\mathrm{Ca}^{2+}\right]_{i}$ transient recovery phase in the coupled donor-derived myocytes was very similar to that seen for uncoupled donor-derived myocytes during field stimulation: values for $t_{90-50 \%}, t_{50-10 \%}, C a T_{50 \%}$, and $C_{a} T_{90 \%}$ were $79 \pm 8.9$, $123 \pm 7.6,141 \pm 11.3$, and $264 \pm 17.6 \mathrm{~ms}$, respectively (stimulation at $2 \mathrm{~Hz} ; 12$ cells, distributed among four recipient hearts, were analyzed). The heterogeneity in $\left[\mathrm{Ca}^{2+}\right]_{\mathrm{i}}$ transient duration observed during spontaneous depolarization (about $2 \mathrm{~Hz}$ ) persisted during point stimulation at $4 \mathrm{~Hz}$ (data not shown). A total of 177 donorderived myocytes exhibiting $\left[\mathrm{Ca}^{2+}\right]_{\mathrm{i}}$ transients synchronous with those in neighboring cardiomyocytes during spontaneous sinus rhythm have been imaged. These cells were distributed among 27 different animals and in grafts ranging from 11 to 106 days old. Thus, on average fewer than seven coupled donor-derived myocytes per heart were detected in the areas amenable to image analysis.

Functionally coupled donor-derived myocytes retain characteristics of skeletal muscle cells. The $\left[\mathrm{Ca}^{2+}\right]_{\mathrm{i}}$ transient shape and recoveryphase heterogeneity suggested that coupled donor-derived myocytes retain functional attributes characteristic of skeletal muscle. To further explore this point, the $\left[\mathrm{Ca}^{2+}\right]_{i}$ response to incremental increases in stimulation rate was monitored in the coupled donor-derived myocytes and neighboring host cardio- myocytes using line-scan analysis. The line scan traversed three juxtaposed myocytes (Figure 4A, white line); cell 1 was a coupled donorderived myocyte (EGFP positive), and cells 2 and 3 were host cardiomyocytes (EGFP negative). The cells were subjected to field stimulation at frequencies ranging from $2 \mathrm{~Hz}$ to $7 \mathrm{~Hz}$. Examination of the resulting stacked line scans (Figure 4B) and the integrated traces (Figure 4C) revealed that the coupled donorderived myocyte developed tetanus. In contrast, tetanus did not develop with increased pacing rates in the neighboring host cardiomyocytes (rather, a 2:1 stimulus-response block eventually occurred). These observations suggested that coupled donor-derived myocytes retain functional characteristics of skeletal muscle cells. It is well documented that sustained rapid pacing of isolated mouse skeletal muscle fibers induced fatigue, which was manifest by marked decreases in both developed tension and $\left[\mathrm{Ca}^{2+}\right]_{i}$ transient amplitude $(15,16)$. Fatigue was also readily induced in myoblast-derived skeletal myocytes during sustained pacing at $10 \mathrm{~Hz}$ (Figure 4D).

Low levels of cellular fusion between donor skeletal myoblasts and host cardiomyocytes. Recent studies have demonstrated that a number of multipotent stem cells can fuse with host cardiomyocytes following systemic delivery $(17,18)$. To determine whether skeletal myoblasts could fuse with host cardiomyocytes, myoblasts prepared from $A C T-E G F P$ mice were injected into the hearts of myosin heavy chain- $n L A C$ (MHC- $n L A C)$ transgenic mice. The $M H C-n L A C$ reporter gene targets expression of a nLAC reporter exclusively to cardiomyocytes $(11,19)$; consequently, cardiomyocyte nuclei stain blue when histologic sections from $\mathrm{MHC}$ $n L A C$ mice are incubated with 5-bromo-4-chloro-3-indolyl- $\beta$-Dgalactoside (X-GAL, a chromogenic substrate for $\beta$-gal). Fusion events between donor EGFP-expressing myoblasts and host nLAC-expressing cardiomyocytes can thus easily be identified by the presence of blue nuclei in cells with green fluorescent cytoplasm following X-GAL staining.

To screen for cell fusion events, hearts from $M H C-n L A C$ mice that received ACT-EGFP myoblast transplants were harvested, sectioned, stained with X-GAL, and visualized under bright field illumination. Cardiomyocyte nuclei at the graft/myocardium border were readily identified by the presence of blue nuclear staining (Figure 5, A-C). Examination of the same microscopic fields under fluorescence illumination (Figure 5, D-F, respectively) revealed that some myocytes with nuclear $\beta$-gal activity also exhibited cytoplasmic EGFP fluorescence (arrows). The presence of cells with green cytoplasm and blue nuclei is indicative of 


\section{Table 1}

Properties of $\left[\mathrm{Ca}^{2+}\right]_{i}$ transients in functionally coupled, donorderived myocytes and bordering host cardiomyocytes

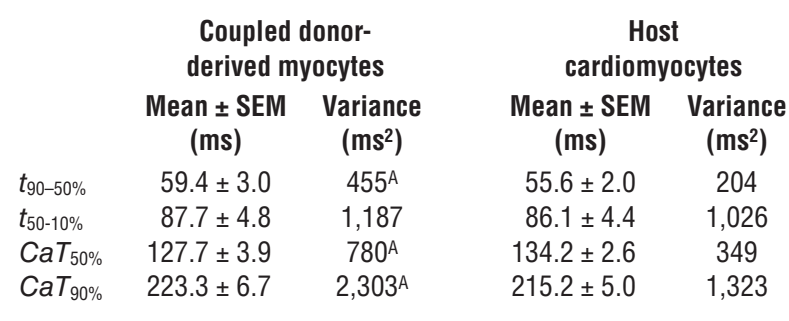

AP $<0.05$ vs. host cardiomyocytes by $F$ test. Fifty-two EGFP-positive donor-derived myocytes and their neighboring host cardiomyocytes, distributed among 17 hearts, were analyzed. The rate at which $\left[\mathrm{Ca}^{2+}\right]_{\mathrm{i}}$ transients were recorded averaged $2.0 \pm 0.03 \mathrm{~Hz}$.

fusion events between donor skeletal myoblasts and host cardiomyocytes. Given the typical dimensions of adult cardiomyocytes and myoblast-derived skeletal myocytes, and given that the sections analyzed were $10 \mu \mathrm{m}$ thick, the presence of a blue nucleus within a green cytoplasm cannot result from simple overlay of two individual cells. Cell fusion events were detected only at the graft/myocardium border. A total of 83 fusion events were found in six hearts harvested between 27 and 50 days following transplantation. This represents on average less than one myoblastcardiomyocyte fusion event per graft-containing section.

To further characterize the fused cells, sections prepared from $M H C-n L A C$ hearts that received ACT-EGFP skeletal myoblast grafts were processed for anti-connexin 43 immune histologic analyses. Connexin 43 immune reactivity was readily detected between neighboring host cardiomyocytes, as evidenced by punctate red signal at the junctional borders between myocytes with blue nuclei and nonfluorescent cytoplasm (Figure 6, A-C). In contrast, no connexin 43 immune reactivity was detected between donor-derived myocytes within the center of the graft, as evidenced by the absence of punctate red signal at the junctional borders of myocytes with green fluorescent cytoplasm and nonblue nuclei (Figure 6, D-F; diffuse red autofluorescence was frequently observed in the donor-derived myocyte grafts). This result was expected, given the absence of functional coupling between these cells (see above). Examination of cells arising from fusion events (cells with blue nuclei and green fluorescent cytoplasm) revealed the presence of connexin 43 immune reactivity (punctate red signal) at the junctional borders of neighboring host cardiomyocytes (cells with blue nuclei and nonfluorescent cytoplasm; see Figure 6, G-L). Thus, cells arising from fusion events between the donor skeletal myoblasts and host cardiomyocytes retained molecular attributes required for electrical communication with the host myocardium.

\section{Discussion}

The data presented here revealed that while both spontaneous and remote point stimulation-evoked $\left[\mathrm{Ca}^{2+}\right]_{\mathrm{i}}$ transients were observed to occur synchronously in host cardiomyocytes, they were absent in the vast majority of donor-derived myocytes. These observations were in good agreement with previous studies demonstrating functional dissociation of skeletal myocyte grafts from the recipient myocardium (2-4). Functional isolation could have resulted from a decrease in cell-to-cell coupling and/ or from an intrinsic decrease in donor-derived myocyte excitability. Although previous studies found no immune cytologic evidence for the presence of junctional complexes (3) and no evidence for dye transfer (4), they did not rule out the possibility of atypical donor-derived myocyte-cardiomyocyte coupling. For example, heterotypic junctional complexes (20) or fibroblastmyocyte coupling (21) would not have been detected by these assays. The data presented here unequivocally demonstrate that the preponderance of engrafted donor-derived myocytes were functionally isolated from the host myocardium, even when they were in direct physical contact with host cardiomyocytes.

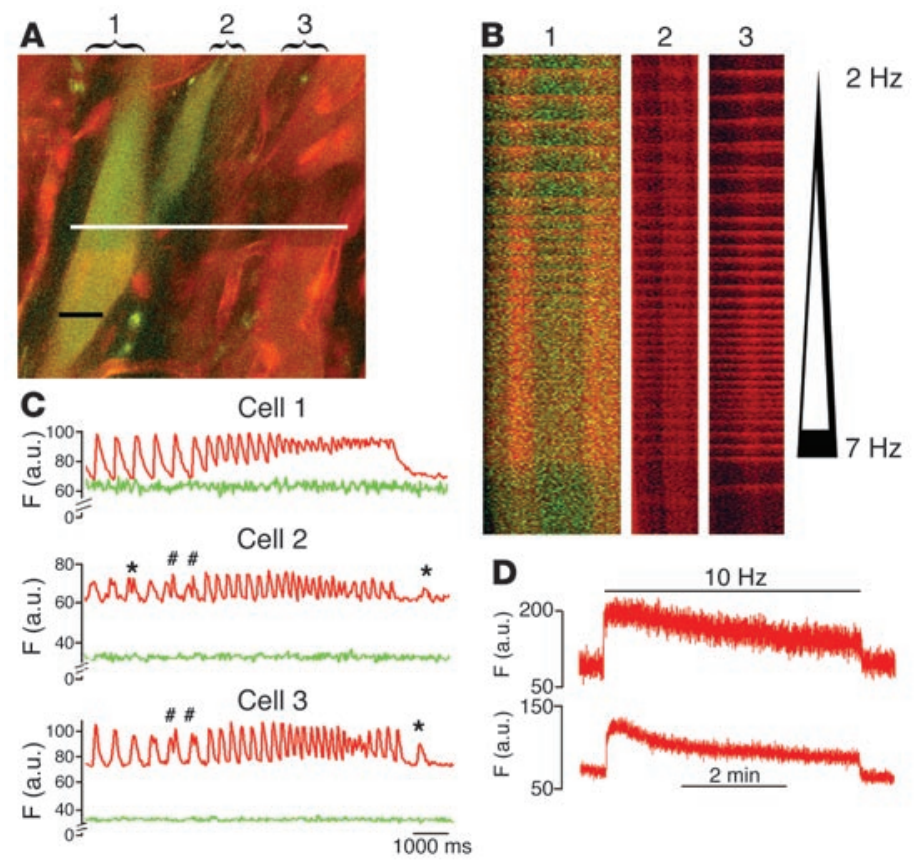

\section{Figure 4}

Incremental increases in the frequency of field stimulation cause development of $\left[\mathrm{Ca}^{2+}\right]_{i}$ transients (tetanus) in a coupled donorderived, EGFP-expressing myocyte but not in neighboring cardiomyocytes. (A) Full frame-mode image of a spontaneous $\left[\mathrm{Ca}^{2+}\right]_{i}$ transient in a rhod-2-loaded heart at 16 days after transplantation of ACT-EGFP skeletal myoblasts; rhod-2 (red) and EGFP (green) signals were superimposed. The white bar marks the position of the line scan-mode data acquisition. Scale bar: $10 \mu \mathrm{m}$. (B) Stacked line-scan images of the regions in $\mathbf{A}$ indicated by the white line for cells numbered 1 (a coupled, donor-derived myocyte), 2, and 3 (cardiomyocytes). The pacing rate is indicated. (C) Spatially integrated traces of the changes in rhod-2 (red) and EGFP (green) fluorescence for cells 1,2 , and 3 . Note that tetanus develops in the coupled donor-derived myocyte, but not in the cardiomyocytes. Identical results were observed when an additional 9 coupled EGFP-expressing myocytes were analyzed. * $\mathrm{Ca}^{2+}$ waves; "dualpeak elevations of $\left[\mathrm{Ca}^{2+}\right]_{i}$ transients due to superimposition of spontaneous and field stimulation-induced transients. (D) Sustained rapid stimulation induced a decline of $\left[\mathrm{Ca}^{2+}\right]_{i}$ in in vitro-differentiated skeletal myocytes. Line-scan images from two skeletal myocytes during rest and during continuous field stimulation at $10 \mathrm{~Hz}$ for 5 minutes were obtained, and spatially averaged rhod2 intensities were plotted as function of time. Rapid stimulation causes an initial elevation of $\left[\mathrm{Ca}^{2+}\right]$ i followed by a slow decay. 
A

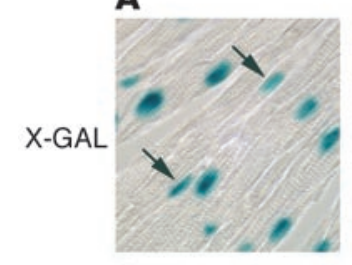

D

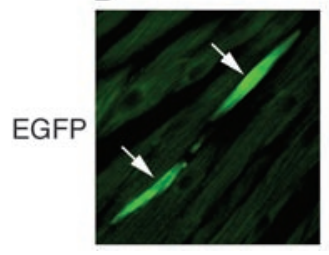

B

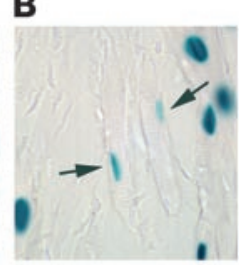

E

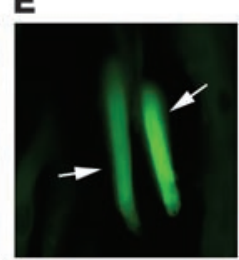

C

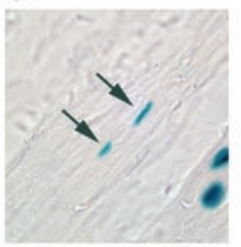

$\mathbf{F}$

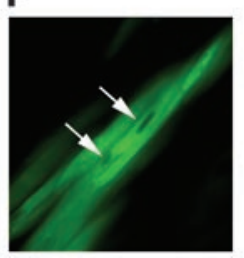

Figure 5

Donor skeletal myoblasts can fuse with host cardiomyocytes. (A-C) Bright field photomicrographs of $10-\mu \mathrm{m}$ sections from MHC-nLAC hearts transplanted with ACT-EGFP skeletal myoblasts. $\beta$-Gal-positive cardiomyocyte nuclei at the graft/myocardium border are readily seen. Hearts were harvested between 46 and 49 days after transplantation. (B-F) Fluorescent photomicrographs from the same fields depicted in A-C. Arrows identify the myocytes with blue nuclear staining and green fluorescent cytoplasm.

In light of these results, the observation that a small number of the donor-derived myocytes exhibited spontaneous or stimulation-induced calcium transients in synchrony with their neighboring host cardiomyocytes was quite intriguing. These cells were observed only infrequently, and were always located at or near the graft/myocardium border. The coupled donor-derived myocytes developed tetanus upon rapid stimulation, indicating that they retained functional properties characteristic of skeletal myotubes. This view was also supported by the heteroge-

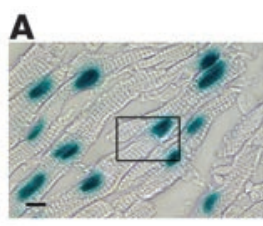

D

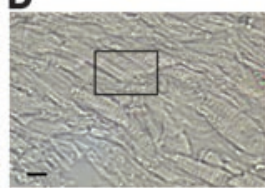

G

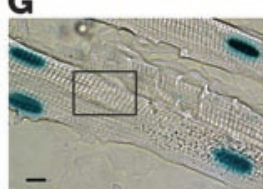

$\mathbf{J}$

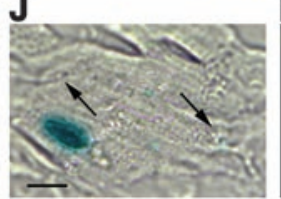

B

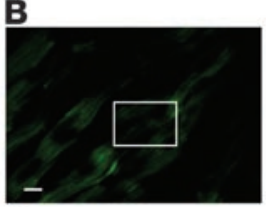

$\mathbf{E}$

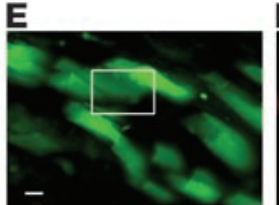

H

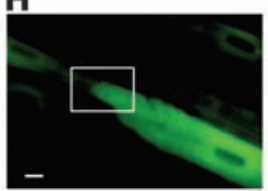

K

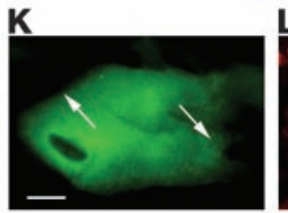

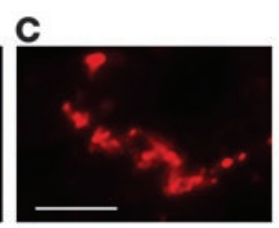

$\mathbf{F}$

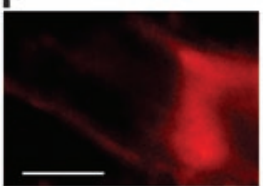

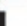

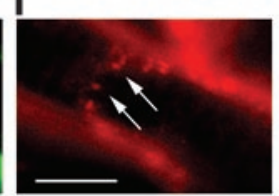

$\mathbf{L}$

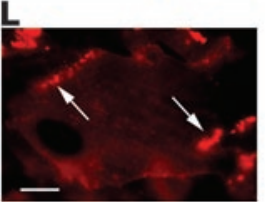

neity observed in the recovery phase of the $\left[\mathrm{Ca}^{2+}\right]_{\mathrm{i}}$ transients in donor-derived myocytes in situ as well as in in vitro-differentiated skeletal myocytes.

Although a considerable number of both uncoupled and coupled donor-derived myocytes were imaged in this study, it is important to note that these numbers did not reflect the relative frequency of these cell types. Indeed, donor-derived myocytes with synchronized transients constituted only a very small percentage of the total number of engrafted EGFP-positive myocytes, and were located only at the graft/host myocardium border. Moreover, no donor-derived myocytes located within grafts exhibited $\left[\mathrm{Ca}^{2+}\right]_{i}$ transients. Thus, the vast majority of engrafted EGFP-expressing myocytes were functionally isolated from the host myocardium. Because we could not quantitate the total number of EGFP-expressing cells in individual hearts (due to intrinsic limitations of tissue sampling and penetration with the TPME imaging system), it was impossible to determine the precise frequency at which coupled donor-derived myocytes appeared. Nonetheless it was very clear that the coupled EGFPexpressing donor-derived myocytes were too few in number to contribute significantly to global cardiac function.

The observation that transplantation of EGFP-expressing myoblasts into the hearts of MHC-nLAC mice resulted in the formation of myocytes with green fluorescent cytoplasm and blue nuclei following X-GAL staining indicated that donor myoblasts also fused with host cardiomyocytes. Several studies have demonstrated that donor hematopoietic stem cells fused with host hepatocytes following in vivo delivery $(22,23)$. In addition, stem cells derived from the bone marrow (17) or from the adult heart (18) were observed to fuse with host cardiomyocytes following delivery into lethally irradiated or infarcted recipients, respectively. Moreover, Murry and colleagues have recently demonstrated fusion events between skeletal myoblasts and cardiomyocytes in vivo using genetic approaches similar to those used here (24). The observation that cells arising from skeletal myocyte-cardiomyo-

\section{Figure 6}

Connexin43 immune reactivity between cells arising from myoblastcardiomyocyte fusion events and host cardiomyocytes. Ten-micron sections prepared from MHC-nLAC hearts transplanted with $A C T$ EGFP skeletal myoblasts were stained with X-GAL and reacted with an anti-connexin43 $\mathrm{Ab}$, followed by a rhodamine-conjugated secondary $\mathrm{Ab} .(\mathbf{A}-\mathbf{C})$ Connexin43 is present at the junction of host cardiomyocytes. Host cardiomyocytes are identified by blue nuclear staining (A) and the absence of EGFP fluorescence (B). (C) A higher-magnification image of connexin43 immune reactivity in the boxed region is shown (red signal). (D-F) Connexin43 is absent between donor-derived skeletal myoblasts. Donor-derived myocytes are identified by the absence of blue nuclear staining (D) and the presence of green fluorescence (E). (F) A higher-magnification image of the boxed region after staining for connexin43 immune reactivity. Note the absence of punctate red signal. (G-I) Connexin43 is present at the junction between cells derived from myocyte-cardiomyocyte fusion events and host cardiomyocytes. Cells arising from myocyte-cardiomyocyte fusion events are identified by the presence of blue nuclear staining (G) and EGFP fluorescence $(\mathbf{H})$. Host cardiomyocytes are identified by the presence of nuclear $\beta$-gal activity and the absence of EGFP fluorescence. (I) A higher-magnification image of connexin43 immune reactivity in the boxed region. Arrows mark punctate red signal, indicative of the presence of connexin43 (and hence, the presence of gap junctions). ( $\mathbf{J}-\mathbf{L}$ ) Additional example of connexin43; arrows indicate the position of the gap junctions. Scale bars: $10 \mu \mathrm{m}$. 
cyte fusion events expressed connexin 43 raised the intriguing possibility that these were the functionally coupled donor-derived cells detected by TPME imaging. This view was further supported by the similarity in the relative frequency of occurrence as well as the anatomical distribution of the coupled donor-derived myocytes compared with the cells arising from fusion events. However, the experimental approaches used here did not rule out the possibility that atypical mechanisms of donor-derived skeletal myocyte-host cardiomyocyte coupling might also have contributed to the functional coupling observed via TPME imaging.

The heterogeneity in $\left[\mathrm{Ca}^{2+}\right]_{i}$ transient duration observed in the coupled donor-derived myocytes resulted in the momentary presence of myocytes with high $\left[\mathrm{Ca}^{2+}\right]_{\mathrm{i}}$ juxtaposed with and coupled to cells with normal $\left[\mathrm{Ca}^{2+}\right]_{\mathrm{i}}$. Such microheterogeneity in $\left[\mathrm{Ca}^{2+}\right]_{\mathrm{i}}$ has been implicated in the arrhythmogenesis in ischemic myocardium at the infarct border zone $(25,26)$. Ventricular tachyarrhythmias were observed, albeit infrequently, in some patients following myoblast transplantation $(5,6)$. However it was not clear whether the small number of coupled donorderived myocytes that displayed heterogeneity in $\left[\mathrm{Ca}^{2+}\right]_{\mathrm{i}}$ transient recovery-phase duration in our study would have comprised a sufficient cellular mass to trigger arrhythmias (27). Other factors could easily have contributed to the arrhythmias observed in the clinical studies, including the interdigitation of noncoupled donor-derived myocytes and host cardiomyocytes at the graft/ myocardium border (which mimics electrophysiological heterogeneity observed at the infarct border zone; see ref. 28), as well as transient donor cell-induced ischemia (as was recently observed following mesenchymal stem cell transplantation; see ref. 29). Previous studies failed to detect arrhythmias in mouse hearts with skeletal myoblast grafts during surface electrocardiogram analyses (2). Given the high spontaneous rate of intact mouse hearts (30) it is likely that the coupled donor-derived myocytes were fatigued in vivo. Caveats pertaining to source/sink mismatch, border zone heterogeneity, and ischemia notwithstanding, it is highly likely that if fatigued, coupled donor-derived myocytes would not have been able to induce arrhythmia.

In summary, the data presented here indicated that the preponderance of donor-derived myocytes resulting from the intracardiac delivery of skeletal myoblasts remained functionally isolated from the host myocardium, and retained functional properties characteristic of in vitro-differentiated skeletal myocytes. However, a small percentage of the donor-derived myocytes were functionally coupled to the host myocardium, and a significant fraction of these exhibited heterogeneity in the duration of the $\left[\mathrm{Ca}^{2+}\right]_{\mathrm{i}}$ transient recovery phase. Myoblast-cardiomyocyte fusion was also observed. The presence of gap junctional complexes, the relative frequency of occurrence, and the anatomical distribution of the cells arising from myoblast-cardiomyocyte fusion events were consistent with the notion that these cells contribute at least in part to the population of coupled donor-derived myocytes identified by TPME imaging.

\section{Methods}

Skeletal myoblast isolation and culture. Skeletal myoblasts were obtained from 8- to 12-week-old heterozygous C57BL/6J mice expressing EGFP under the control of an ACT promoter and cytomegalovirus enhancer (ACT-EGFP; see ref. 10) obtained from The Jackson Laboratory. Myoblast isolation and culture followed the procedure initially described by Konieczny et al. (31, 32). Mice were killed by cervical dislocation, and the hind limbs were skinned and freed of surrounding adipose tissue and fascia. Approximately $1 \mathrm{~g}$ of hind limb muscle was transferred to a dish containing PBS supplemented with $1 \%$ collagenase B, $2.4 \mathrm{U} / \mathrm{ml}$ dispase II (both from Roche Diagnostics Corp.), and $2.5 \mathrm{mmol} / 1 \mathrm{CaCl}_{2}$. The muscle was minced with fine scissors and then incubated at $37^{\circ} \mathrm{C}$. The slurry was intermittently triturated to assist dispersal. After 30 minutes, the cell suspension was passed through sterile $70-\mu \mathrm{m}$ nylon mesh to remove undigested tissue fragments and mature myofibers. The filtered cell suspension was centrifuged at $435 \mathrm{~g}$ for 5 minutes. To remove fibroblasts, the cells were pre-plated on $60-\mathrm{mm}$ plastic tissue culture dishes in $5 \mathrm{ml}$ Ham's F10 medium (Invitrogen Corp.) containing 10\% FBS (HyClone Inc.), $100 \mathrm{U} / \mathrm{ml}$ penicillin, $100 \mu \mathrm{g} / \mathrm{ml}$ streptomycin (Invitrogen Corp.), and $2.5 \mathrm{ng} / \mathrm{ml}$ recombinant human basic fibroblast factor (Invitrogen Corp.). After 2 hours at $37^{\circ} \mathrm{C}$, the nonadherent cells were plated on collagen-coated $60-\mathrm{mm}$ plastic tissue culture dishes at $1 \times 10^{5}$ to $5 \times 10^{5}$ cells per dish (collagen from Roche Diagnostics Corp.). The complete medium was replaced once per day. Subconfluent cultures were passaged every $2-3$ days ( $1: 3$ split) to minimize myogenic differentiation at higher myoblast density. Essentially pure myoblast cultures were obtained within 2-3 weeks. A small aliquot of cells not being used for transplantation was replated on collagen-coated 60-mm dishes and cultured in DMEM containing 5\% horse serum (Invitrogen Corp.) to induce myogenic differentiation. This procedure was performed to ascertain the myogenic potential of cultured myoblasts. For calcium imaging in WT myotubes, skeletal myoblasts from nontransgenic C57BL/6J mice were isolated and cultured in the same way as described above. Myotubes were used between 7 and 10 days after the first exposure to differentiating medium.

TPME imaging system and heart preparation. Preparation of the hearts carrying skeletal myoblast grafts and TPME imaging were performed as described previously (9). Images were recorded with an MRC 1024 laser scanning microscope (Bio-Rad Laboratories Inc.) modified for TPME. Illumination for two-photon excitation was provided by a mode-locked Ti:sapphire laser (Spectra-Physics); the excitation wavelength was 810 $\mathrm{nm}$. Hearts were imaged through a Nikon $\times 601.2$ numerical aperture water-immersion lens with a working distance of $200 \mu \mathrm{m}$. Emitted light was collected by two photo-multiplier tubes fitted with narrow bandwidth filters for 560-650 nm and 500-550 nm, respectively. Images were collected at a resolution of $0.43 \mu \mathrm{m} /$ pixel along the $x$ and $y$ axes. For full-frame mode analyses $(512 \times 512$ pixels $)$, hearts were scanned at 1.46 and 0.73 frames per second on horizontal $(x, y)$ planes, and the resulting images were digitized at 8-bit resolution and stored directly on the hard disk. For line-scan mode analyses, hearts were scanned repetitively at a scan speed of $110 \mu \mathrm{m} / \mathrm{ms}$ along a line spanning at least two juxtaposed cardiomyocytes. Line-scan images were then constructed by stacking all lines vertically. Postacquisition analysis was performed using MetaMorph software version 4.6 (Universal Imaging Corp.).

During TPME imaging, hearts were perfused with oxygenated normal Tyrode's solution containing $50 \mu \mathrm{mol} / 1$ cytochalasin D to eliminate contraction-induced movement. Unless otherwise indicated, all records were made during spontaneous sinus rhythm, which occurred at a rate of approximately $2 \mathrm{~Hz}$ under the conditions used. Point stimulation was performed as described previously $(8,9)$. Electrical field stimulation of Langendorff-perfused hearts was performed by positioning the heart between a pair of loop-shaped platinum wires placed approximately $1 \mathrm{~cm}$ apart, with the current flowing roughly perpendicular to the longitudinal axis of the heart. The hearts were stimulated with $60-$ to $100-\mathrm{V}$ pulses of 1 - to $2-\mathrm{ms}$ duration using a Grass-Telefactor model SD9 stimulator.

The fluorescent profiles of $\left[\mathrm{Ca}^{2+}\right]_{\mathrm{i}}$ transients were obtained by averaging the line-plot data of sequential line scans. For determination of the time course of $\left[\mathrm{Ca}^{2+}\right]_{\mathrm{i}}$ decay, rhod-2 fluorescence was normalized to the 
difference between peak and baseline intensity, and intervals from $90 \%$ to $50 \%\left(t_{90-50 \%}\right)$ and $50 \%$ to $10 \%\left(t_{50-10 \%}\right)$ were calculated. $\left[\mathrm{Ca}^{2+}\right]_{\mathrm{i}}$ transient duration at $50 \%\left(\mathrm{Ca}_{50 \%}\right)$ and $90 \%$ recovery $\left(\mathrm{CaT}_{90 \%}\right)$ was determined by measuring the time intervals from the onset of the transient $(10 \%$ above average baseline values) to the point when $\left[\mathrm{Ca}^{2+}\right]_{i}$ returns to within $50 \%$ and $90 \%$ of resting levels, respectively.

Measurements of $\left[\mathrm{Ca}^{2+}\right]_{i}$ in single skeletal myotubes in culture. For the measurements of $\left[\mathrm{Ca}^{2+}\right]_{\mathrm{i}}$ transients in response to action potentials, myotubes were loaded with rhod-2. The cells were washed free of the culture medium by continuous superfusion with normal Tyrode's solution for 15 minutes at $2 \mathrm{ml} / \mathrm{min}$. They were then exposed to a solution containing 10 $\mu \mathrm{mol} / 1 \mathrm{rhod}-2$ plus $0.02 \%$ (wt/vol) Pluronic F127 (Molecular Probes Inc.) in normal Tyrode's solution for 20 minutes at room temperature. Cells were subsequently returned to superfusion with dye-free solution for 20 minutes at $2 \mathrm{ml} / \mathrm{min}$ before experimentation. Cells in $60-\mathrm{mm}$ dishes were placed on the stage of an upright microscope modified for two-photon illumination (LSM 510 META, Carl Zeiss Inc.) and imaged through a $\times 400.8$ NA water dipping objective (Achroplan IR, Carl Zeiss Inc.). Twophoton illumination was provided by a mode-locked Ti:sapphire laser (Spectra-Physics) tuned to a center wavelength of $810 \mathrm{~nm}$. Pulse duration and pulse repetition rate were $100 \mathrm{fs}$ and $83 \mathrm{MHz}$, respectively. Emitted light was collected by a photomultiplier fitted with a narrow-bandwidth filter for 560 to $650 \mathrm{~nm}$. To induce action potential-evoked $\left[\mathrm{Ca}^{2+}\right]_{\mathrm{i}}$ transients, myotubes were field stimulated via 1 -ms square wave pulses with approximately 1.2-fold threshold amplitude. The stimuli were delivered by a programmable stimulator (SD9, Grass-Telefactor) via a pair of platinum wire electrodes held in position by a micromanipulator. Cells were imaged in line-scan mode. A single line across the entire cell width was repeatedly scanned at a frequency of $325 \mathrm{~Hz}$, and composite line-scan images were constructed by stacking scan lines vertically. Fluorescence signals were digitized at 8-bit resolution and stored on the computer's hard disk for offline analysis. All experiments were performed at room temperature. For determination of $\mathrm{Ca} T_{50 \%}, \mathrm{Ca} T_{90 \%}, t_{90-50 \%}$, and $t_{50-10 \%}$ (see above), signals from three consecutive transients were averaged.

Skeletal myoblast transplantation. Immediately before injection, myoblasts were harvested with $0.05 \%$ trypsin/EDTA in PBS at $37^{\circ} \mathrm{C}$ and washed three times with PBS. They were then injected directly into the ventricular myocardium of adult syngeneic C57BL/6J mice (The Jackson Laboratory) under open-heart surgery as described (33). One hundred thousand cells were injected in a volume of 2-3 $\mu \mathrm{l}$ using a plastic syringe fitted with a 27 gauge needle. All animal experiments were approved by the Institutional Animal Care and Use Committee, Indiana University School of Medicine.
Detection of donor-host fusion in vivo. To determine whether transplanted skeletal myoblasts fuse spontaneously with host cardiomyocytes in vivo, skeletal myoblasts from ACT-EGFP mice were injected into MHC-nLAC mice (11). MHC-nLAC mice use the cardiac $\alpha$-MHC promoter to target expression of a nuclear-localized $\beta$-gal (nLAC) reporter gene. Consequently, fused cells could be detected by staining with X-GAL (Roche Diagnostics Corp.) followed by epifluorescence microscopy of thin sections from $M H C-n L A C$ hearts bearing ACT-EGFP skeletal myoblast grafts. After 16-50 days, mice were sacrificed by cervical dislocation, the hearts were excised, the ascending aorta was cannulated with an 18-gauge needle, and the hearts were retrogradely perfused with $1 \%$ paraformaldehyde and $1 \%$ cacodylic acid, pH 7.4, at room temperature. Hearts were cryoprotected and sectioned at $10 \mu \mathrm{m}$. $\beta$-Gal activity was detected by staining with X-GAL. For connexin43 immune histology, hearts were processed and stained with X-GAL as described above. Sections were then incubated with $0.2 \%$ Triton X-100 (Sigma-Aldrich) in PBS for 1 hour, followed by 30 minutes of blocking with $2 \%$ BSA. Sections were incubated for 12 hours with primary $\mathrm{Ab}$ in PBS supplemented with $2 \% \mathrm{BSA}$ and $10 \%$ normal goat serum. A commercially available monoclonal anti-connexin $43 \mathrm{Ab}$ (raised against residues 252-270 of rat connexin43; MAB 3068, Chemicon International) was used at 1:250 dilution. Sections were subsequently reacted with rhodamine-conjugated rabbit anti-mouse IgG secondary $\mathrm{Ab}$ (AP160R, Chemicon) at 1:50 dilution. All incubation steps were performed at room temperature, and between all incubation steps the slides were thoroughly washed with PBS three times for 5 minutes each.

Statistical analyses. Data are presented as mean \pm SEM. The results were analyzed using the two-tailed $F$ test and the Student's $t$ test. Differences were considered significant at $P<0.05$.

\section{Acknowledgments}

We thank the Indiana Center for Biological Microscopy for assistance. This work was supported by grants from the National Heart, Lung, and Blood Institute (NHLBI).

Received for publication March 15, 2004, and accepted in revised form July 22, 2004.

Address correspondence to: Loren J. Field, Herman B. Wells Center for Pediatric Research, Indiana University School of Medicine, R4 Building, Room W376, 1044 West Walnut Street, Indianapolis, Indiana 46202-5225, USA. Phone: (317) 274-5085; Fax: (317) 2748679; E-mail: ljfield@iupui.edu.
1. Dowell, J., Rubart, M., Pasumarthi, K.B.S., Soonpaa, M.H., and Field, L.J. 2003. Myocyte and myogenic stem cell transplantation in the heart. Cardiovasc. Res. 58:336-350.

2. Koh, G.Y., Klug, M.G., Soonpaa, M.H., and Field, L.J. 1993. Differentiation and long-term survival of C2C12 myoblast grafts in heart. J. Clin. Invest. 92:1548-1554.

3. Murry, C.E., Wiseman, R.W., Schwartz, S.M., and Hauschka, S.D. 1996. Skeletal myoblast transplantation for repair of myocardial necrosis. J. Clin. Invest. 98:2512-2523.

4. Leobon, B., et al. 2003. Myoblasts transplanted into rat infarcted myocardium are functionally isolated from their host. Proc. Natl. Acad. Sci. U. S. A. 100:7808-7811.

5. Menasche, P., et al. 2003. Autologous skeletal myoblast transplantation for severe postinfarction left ventricular dysfunction. J. Am. Coll. Cardiol. 41:1078-1083.

6. Smits, P.C., et al. 2003. Catheter-based intramyocardial injection of autologous skeletal myoblasts as a primary treatment of ischemic heart failure: clinical experience with six-month followup. J. Am. Coll. Cardiol. 42:2063-2069.

7. Pagani, F.D., et al. 2003. Autologous skeletal myoblasts transplanted to ischemia-damaged myocardium in humans. Histological analysis of cell survival and differentiation. J. Am. Coll. Cardiol. 41:879-888.

8. Rubart, M., Wang, E., Dunn, K.W., and Field, L.J. 2003. Two-photon molecular excitation imaging of $\mathrm{Ca}^{2+}$ transients in Langendorff-perfused mouse hearts. Am. J. Physiol. Cell Physiol. 284:C1654-C1668.

9. Rubart, M., et al. 2003. Physiological coupling of donor and host cardiomyocytes after cellular transplantation. Circ. Res. 92:1217-1224.

10. Okabe, M., Ikawa, M., Kominami, K., Nakanishi, T., and Nishimune, Y. 1997. 'Green mice' as a source of ubiquitous green cells. FEBS Lett. 407:313-319.

11. Soonpaa, M.H., Koh, G.Y., Klug, M.G., and Field, L.J. 1994. Formation of nascent intercalated disks between grafted fetal cardiomyocytes and host myocardium. Science. 264:98-101.
12. Liu, Y., Kranias, E.G., and Schneider, M.F. 1997. Regulation of $\mathrm{Ca}^{2+}$ handling by phosphorylation status in mouse fast- and slow-twitch skeletal muscle fibers. Am. J. Physiol. 273:C1915-C1924.

13. Garcia, J., and Beam, K.G. 1994. Measurement of calcium transients and slow calcium current in myotubes. J. Gen. Physiol. 103:107-123.

14. Flucher, B.E., and Andrews, S.B. 1993. Characterization of spontaneous and action potential-induced calcium transients in developing myotubes in vitro. Cell Motil. Cytoskeleton. 25:143-157.

15. Westerblad, H., and Allen, D.G. 1991. Changes of myoplasmic calcium concentration during fatigue in single mouse muscle fibers. J. Gen. Physiol. 98:615-635.

16. Westerblad, H., Lee, J.A., Lannergren, J., and Allen, D.G. 1991. Cellular mechanisms of fatigue in skeletal muscle. Am. J. Physiol. 261:C195-C209.

17. Alvarez-Dolado, M., et al. 2003. Fusion of bonemarrow-derived cells with Purkinje neurons, cardiomyocytes and hepatocytes. Nature. 425:968-973.

18. Oh, H., et al. 2003. Cardiac progenitor cells from 
adult myocardium: homing, differentiation, and fusion after infarction. Proc. Natl. Acad. Sci. U. S. A. 100:12313-12318.

19. Soonpaa, M.H., and Field, L.J. 1997. Assessment of cardiomyocyte DNA synthesis in normal and injured adult mouse hearts. Am. J. Physiol. 272:H220-H226.

20. Martinez, A.D., Hayrapetyan, V., Moreno, A.P. and Beyer, E.C. 2002. Connexin 43 and connexin 45 form heteromeric gap junction channels in which individual components determine permeability and regulation. Circ. Res. 90:1100-1107.

21. Gaudesius, G., Miragoli, M., Thomas, S.P., and Rohr, S. 2003. Coupling of cardiac electrical activity over extended distances by fibroblasts of cardiac origin. Circ. Res. 93:421-428.

22. Vassilopoulos, G., Wang, P., and Russell, D.W. 2003. Transplanted bone marrow regenerates liver by cell fusion. Nature. 422:901-904.

23. Wang, X., et al. 2003. Cell fusion is the principal source of bone-marrow-derived hepatocytes
Nature. 422:897-901.

24. Reinecke, H., Minami, E., Poppa, V., and Murry, C.E. 2004. Evidence for fusion between cardiac and skeletal muscle cells. Circ. Res. 94:E56-E60.

25. Lamont, C., Luther, P.W., Balke, C.W., and Wier, W.G. 1998. Intercellular $\mathrm{Ca}^{2+}$ waves in rat heart muscle. J. Physiol. 512:669-676.

26. Miura, M., Boyden, P.A., and ter Keurs, H.E. 1999. $\mathrm{Ca}^{2+}$ waves during triggered propagated contractions in intact trabeculae. Determinants of the velocity of propagation. Circ. Res. 84:1459-1468.

27. Rohr, S., Kucera, J.P., Fast, V.G., and Kleber, A.G. 1997. Paradoxical improvement of impulse conduction in cardiac tissue by partial cellular uncoupling. Science. 275:841-844.

28. Yao, J.A., et al. 2003. Remodeling of gap junctional channel function in epicardial border zone of healing canine infarcts. Circ. Res. 92:437-443.

29. Vulliet, P.R., Greeley, M., Halloran, S.M., MacDonald, K.A., and Kittleson, M.D. Coronary arterial injection of mesenchymal stromal cells produces myocardial ischemia/infarction in dogs. Lancet. 363:783-784.

30. Steinhelper, M.E., Cochrane, K.L., and Field, L.J. 1990. Hypotension in transgenic mice expressing atrial natriuretic factor fusion genes. Hypertension. 16:301-307.

31. Konieczny, S.F., McKay, J., and Coleman, J.R. 1982 Isolation and characterization of terminally differentiated chicken and rat skeletal muscle myoblasts. Dev. Biol. 91:11-26.

32. Smith, T.H., Block, N.E., Rhodes, S.J., Konieczny, S.F., and Miller, J.B. 1993. A unique pattern of expression of the four muscle regulatory factor proteins distinguishes somitic from embryonic, fetal and newborn mouse myogenic cells. Development. 117:1125-1133

33. Koh, G.Y., Soonpaa, M.H., Klug, M.G., and Field, L.J. 1993. Long-term survival of AT-1 cardiomyocyte grafts in syngeneic myocardium. Am. J. Physiol. Heart Circ. Physiol. 264:H1727-H1733. 